\title{
Impact of irregular marital cohabitation on quality of life and sexual dysfunction in infertile men from upper Egypt
}

\author{
Emad Abdelrhim Taha ${ }^{1}$, Mohamed Sabry ${ }^{2}$, Islam Fathy Soliman Abdelrahman ${ }^{3}$, Hossam Elktatny ${ }^{4}$, Ahmed Hosny ${ }^{5}$ \\ 'Department of Dermatology, Venereology and Andrology, Faculty of Medicine, Assiut University, Asyut; ${ }^{2}$ Department of Obstetrics and Gynecology, \\ Faculty of Medicine, Sohag University, Sohag; ${ }^{3}$ Department of Andrology, Faculty of Medicine, Cairo University, Cairo; ${ }^{4}$ Department of Obstetrics and \\ Gynecology, Faculty of Medicine, Al Azhar Assiut University, Asyut; ${ }^{5}$ Department of Dermatology and Andrology, Faculty of Medicine, Helwan \\ University, Helwan, Egypt
}

Objective: Irregular patterns of marital cohabitation are a common problem in upper Egypt due to employment conditions. The objective of this study was to investigate the effect of irregular marital cohabitation on the quality of life and sexual function of infertile men.

Methods: In total, 208 infertile men were included and divided into two groups. The first group included 134 infertile men with an irregular pattern of marital cohabitation and the second group included 74 infertile men with a regular pattern of marital cohabitation. All subjects were assessed through a clinical evaluation, conventional semen analysis, the fertility quality of life (FertiQoL) questionnaire, the International Index of Erectile Function (IIEF-5) score, and the premature ejaculation diagnostic tool (PMEDT).

Results: The two groups were compared in terms of conventional semen parameters, FertiQoL, IIEF- 5 score, and PMEDT. Infertile men with an irregular pattern of marital cohabitation had significantly lower subscale and total FertiQoL and IIEF- 5 scores. Additionally, they had significantly higher PMEDT scores. Erectile dysfunction and premature ejaculation were more common in them than in infertile men with a regular pattern of marital cohabitation.

Conclusion: Irregular patterns of marital cohabitation had an adverse effect on quality of life and sexual function in infertile men.

Keywords: Erectile dysfunction; Infertility; Quality of life; Sexual disorders

\section{Introduction}

Infertility is a common public health problem that affects approximately $10 \%$ to $15 \%$ of married couples worldwide [1]. Both infertility and its management options have unfavorable effects on quality of life in affected couples [2,3]. Furthermore, infertile men are at a high-

Received: June 26, 2019 · Revised: September 18, 2019 · Accepted: October 4, 2019 Corresponding author: Ahmed Hosny

Department of Dermatology and Andrology, Faculty of Medicine, Helwan University, Al Sikka Al Hadid Al Gharbeya, Al Masaken Al lqtisadeyah, Helwan, Cairo Governorate 11795, Egypt

Tel:+20-1119500053 Fax:+20-225565820 E-mail:ahmhosny10@yahoo.com

This is an Open Access article distributed under the terms of the Creative Commons Attribution Non-Commercial License (https://creativecommons.org/licenses/by-nc/4.0/) which permits unrestricted non-commercial use, distribution, and reproduction in any medium, provided the original work is properly cited. er risk for sexual dysfunction, as they are more commonly affected by psychopathological health disorders affecting sexual function [4]. Certain occupational patterns, such as shift work, have been shown to negatively influence quality of life by harming the quality of family life, regardless of fertility status [5]. Unemployment and work conditions are lifestyle factors that have also been shown to have a significant impact on quality of life in couples receiving infertility treatment $[6,7]$. Since the majority of solutions for infertility necessitate regular patterns of marital cohabitation-at least around the time of ovulation -work conditions that require men to spend extended periods away from their wives are a further source of stress that may endanger their quality of life and sexual function. Certain geographic areas in upper Egypt have a relative lack of job opportunities, which means that many married men must spend most of the year away from 
their wives in other governorates or countries for work [8]. To the best of our knowledge, no previously published studies have been conducted on such a population to assess the impact of irregular patterns of marital cohabitation on quality of life and sexual function in infertile men. The fertility quality of life (FertiQoL) instrument, the International Index of Erectile Function (IIEF-5), and the premature ejaculation diagnostic tool (PMEDT) questionnaires are simple, noninvasive, and valid tools that have been introduced to objectively assess quality of life and sexual function, especially in infertile men $[9,10]$. This study was conducted to demonstrate the effect of irregular patterns of marital cohabitation on quality of life and sexual function in infertile men.

\section{Methods}

After receiving approval from the Institutional Review Board of the Faculty of Medicine, Assiut University (IRB No. 17100644), this crosssectional study was conducted at infertility clinics in the cities of Sohag and Asyut in upper Egypt, targeting infertile males with oligozoospermia, asthenozoospermia, and/or teratozoospermia. Participants were recruited over the course of 6 months among patients attending outpatient clinics with complaints of infertility.

We excluded infertile men with azoospermia, and those who had not completed 1 year of regular marital cohabitation (to ensure that the couples were affected by infertility). We also excluded male partners in couples also diagnosed with female factor infertility and men with chronic medical or psychiatric illnesses or who had undergone surgery affecting the genitals in the last 3 months. Men with evident hypogonadism or untreated specific genital pathologies, such as varicocele, undescended testes, and penile deformities or fibrosis, were also excluded.

The subjects were divided into two groups: the first included infertile men with an irregular pattern of marital cohabitation due to work conditions, and the second included infertile men with a regular pattern of marital cohabitation. We considered marital cohabitation to be irregular if the husband resided away from the wife for more than 6 months per year or more than 2 weeks per month.

After the study received institutional review board approval and the participants provided informed consent, the patients were evaluated through clinical history-taking, a general physical examination, a genital examination, and conventional semen analysis according to the World Health Organization guidelines [11].

\section{Assessment of fertility-related quality of life using the FertiQoL instrument}

The FertiQoL instrument is a self-reporting questionnaire that assesses quality of life in individuals with fertility problems developed by a collaborative effort of the European Society of Human Reproduction and Embryology, the American Society for Reproductive Medicine, and Merck (Geneva, Switzerland) [12]. The FertiQoL is composed of two modules: the core FertiQoL and the treatment FertiQoL. The Core FertiQoL module consists of 26 items covering four subscales of quality of life (emotional, mind-body, relational, and social). The optional treatment FertiQoL module is composed of 10 items that assess two subscales: environment (six items) and tolerability (four items). The FertiQoL contains a total of six subscales and two scores with a range of 0 to 100 , with higher scores indicating better QoL. We used the printed Arabic version of the FertiQoL, which is available at www.fertiqol.org. The translation from English to Arabic was done by professional translators from Cardiff University.

\section{Assessment of erectile function using the five-item version of the IIEF-5}

We used a validated Arabic-language version of the IIEF-5 [13,14], with five items addressing the relevant domains of male sexual function (erectile dysfunction [ED], orgasmic function, sexual desire, intercourse satisfaction, and overall satisfaction). Each item of the IIEF5 is rated from 1 (very low; almost never or never; or extremely difficult) to 5 (very high; almost always or always; or not difficult), with lower scores indicating more erectile difficulty. A score of $>21$ was considered to denote no ED, while a score $\leq 21$ denoted ED.

\section{Assessment of premature ejaculation using the PMEDT}

Premature ejaculation (PE) was assessed using an Arabic translation of the PMEDT, which has been shown to be satisfactory, feasible, and reliable in numerous validation studies $[9,15]$. It includes five questions: "How difficult is it for you to delay ejaculation?" "Do you ejaculate before you wish?"'"Do you ejaculate with very little stimulation?" "Do you feel frustrated because of ejaculating before you want to?" and "How concerned are you that your time to ejaculation leaves your partner sexually unfulfilled?" Each item has a score of 0 to 4, and the PMEDT is scored by adding the scores for all five items. A score $>10$ is highly suggestive of $\mathrm{PE}$.

\section{Statistical analysis}

Data were analyzed and expressed in tables as mean \pm standard deviation, using SPSS ver. 16.0 (SPSS Inc., Chicago, IL, USA). The unpaired $t$-test and Mann-Whitney test were used to compare parametric and non-parametric variables between the two groups. The chisquare test was used to compare percentages between the two groups. The Pearson correlation test was used to evaluate correlations between quantitative variables. Differences were considered to be statistically significant when $p$-values were equal or less than 0.05 . 
Table 1. Comparison of demographic and clinical variables according to the pattern of marital cohabitation

\begin{tabular}{|c|c|c|c|}
\hline Variable & Group $1(n=134)$ & Group $2(n=74)$ & $p$-value \\
\hline Body mass index $\left(\mathrm{kg} / \mathrm{m}^{2}\right)$ & $19.09-40.56(27.57 \pm 4.95)$ & $19.27-37.55(27.44 \pm 3.25)$ & 0.87 \\
\hline \multicolumn{4}{|l|}{ Infertility type } \\
\hline Primary & $76(51 / 67)$ & $75.7(28 / 37)$ & $0.75^{\mathrm{a})}$ \\
\hline Secondary & $24(13 / 67)$ & $24.3(9 / 37)$ & $0.72^{\mathrm{a})}$ \\
\hline Normal sperm morphology (\%) & $0-50(19.7 \pm 11.9)$ & $2-70(16.24 \pm 14.62)$ & 0.19 \\
\hline Progressive sperm motility (\%) & $0-55(17.61 \pm 11.82)$ & $5-45(21.43 \pm 9.6)$ & 0.09 \\
\hline Sperm viability (\%) & $15-75(41.54 \pm 13.94)$ & $20-67(43.18 \pm 12.09)$ & 0.06 \\
\hline
\end{tabular}

Values are presented as range (mean \pm standard deviation) or percent (number).

Group 1, infertile men with irregular marital cohabitation; Group 2, infertile men with regular marital cohabitation.

${ }^{a}$ Chi-square test.

Table 2. Comparison of FertiQoL, IIEF-5, and PMEDT scores according to the pattern of marital cohabitation

\begin{tabular}{|c|c|c|c|}
\hline Variable & Group 1 & Group 2 & $p$-value \\
\hline \multicolumn{4}{|l|}{ Mean FertiQoL scale } \\
\hline Emotional subscale & $45.27 \pm 7.7$ & $65.20 \pm 12.66$ & $<0.001$ \\
\hline Body mind subscale & $46.45 \pm 8.71$ & $63.85 \pm 14.76$ & $<0.01$ \\
\hline Relational subscale & $38.43 \pm 8.1$ & $60.92 \pm 13.44$ & $<0.001$ \\
\hline Social subscale & $43.09 \pm 8.99$ & $62.84 \pm 13.23$ & $<0.001$ \\
\hline Core FertiQoL & $43.32 \pm 7.72$ & $63.20 \pm 12.41$ & $<0.001$ \\
\hline Optional treatment FertiQoL & $45.45 \pm 10.37$ & $67.77 \pm 9.75$ & $<0.001$ \\
\hline Total FertiQoL & $43.94 \pm 8.22$ & $64.55 \pm 11.17$ & $<0.001$ \\
\hline \multicolumn{4}{|l|}{ Erectile dysfunction } \\
\hline Participants with an abnormal IIEF- 5 score & $61(41 / 67)$ & $13.5(5 / 37)$ & $<0.001^{\text {a) }}$ \\
\hline Mean IIEF-5 score & $15.49 \pm 4.89$ & $21.57 \pm 2.64$ & $<0.01$ \\
\hline \multicolumn{4}{|l|}{ Premature ejaculation } \\
\hline Participants with an abnormal PMEDT score & $46(31 / 67)$ & $8(3 / 37)$ & $<0.001^{\text {a) }}$ \\
\hline Mean PMEDT score & $10.03 \pm 3.57$ & & $<0.01$ \\
\hline
\end{tabular}

Values are presented as mean \pm standard deviation or percent (number).

FertiQoL, fertility quality of life; IIEF-5, International Index of Erectile Function; PMEDT, premature ejaculation diagnostic tool; Group 1, infertile men with irregular marital cohabitation; Group 2, infertile men with regular marital cohabitation.

${ }^{a}$ Chi-square test.

\section{Results}

The study included 208 infertile men from three different governorates in upper Egypt who were divided into two groups. The first included 134 infertile men with an irregular pattern of marital cohabitation and the second included 74 infertile men with a regular pattern of marital cohabitation. The two groups were comparable in terms of mean age, body mass index, infertility duration, distribution of infertility type (primary or secondary), and conventional semen parameters (Table 1).

Infertile men with irregular marital cohabitation had significantly lower subscale and total FertiQoL and IIEF-5 scores. Additionally, they had significantly higher PMEDT scores. ED and PE (indicated by subnormal IIEF-5 and PMEDT scores) were significantly more common in

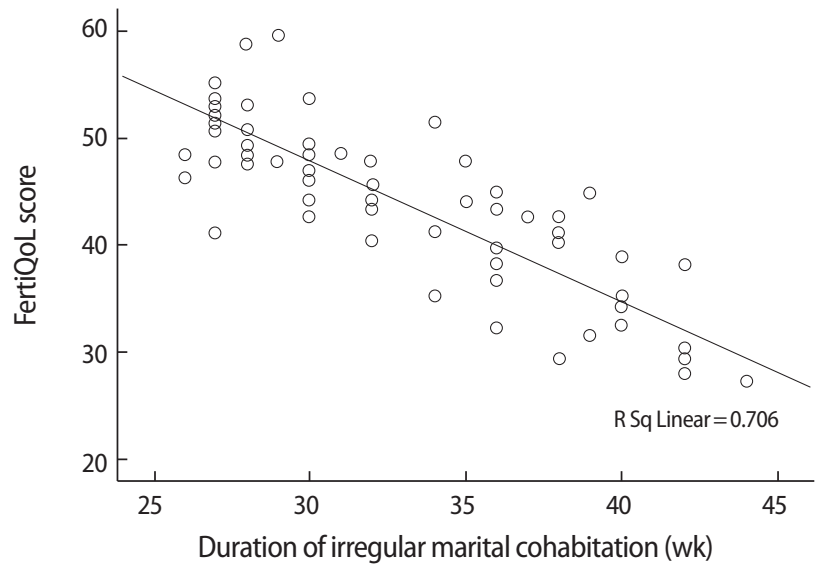

Figure 1. Correlation between the duration of irregular marital cohabitation and total fertility quality of life (FertiQoL) scores. 
infertile men with an irregular pattern of marital cohabitation than in those with a regular pattern of marital cohabitation (Table 2).

Interestingly, significant negative correlations were found between the duration of irregular marital cohabitation (mean number of weeks spent away from home yearly) and scores on the FertiQoL core, treatment, and total scales $(r=-0.529, r=-0.529$, and $r=-0.538$, respectively; $p<0.001$ for each) (Figure 1$)$ and IIIEF-5 scores ( $r=-0.464$, $p<0.001)$. Meanwhile, a significant positive correlation was found between the duration of irregular marital cohabitation and PMEDT scores $(r=0.51, p<0.001)$.

\section{Discussion}

The FertiQoL has been used as a tool to measure the quality of life in infertile women in some studies [16-18]. However, an extensive search of the literature found no studies that investigated the impact of male infertility and irregular marital cohabitation on quality of life among infertile men.

Only four previous studies have used the FertiQoL instrument to investigate factors affecting quality of life in infertile couples [19-22]. A study was conducted in Turkey to identify factors affecting quality of life in 127 infertile couples using the FertiQoL. The researchers concluded that duration of infertility, low income, and younger age of the female partner (less than 30 years) had negative impacts on quality of life [19].

Another study sought to characterize the quality of life of Taiwanese infertile couples. It included 534 infertile subjects (men and women), and the researchers used the core and optional treatment FertiQoL tool to evaluate quality of life. Scores for both the core and treatment FertiQoL were significantly higher in the male partners of infertile couples than in the female partners $(60.63 \pm 14.07 \mathrm{vs}$. $54.39 \pm 13.52$ and $59.13 \pm 12.44$ vs. $56.03 \pm 10.71$, respectively). The core FertiQoL score of infertile men was comparable to our results for men with a regular pattern of marital cohabitation $(64.55 \pm 11.17)$, but higher than that of men with an irregular pattern of marital cohabitation (43.94 \pm 8.22$)$, indicating that irregular marital cohabitation had a negative effect on quality of life among infertile men [22].

The third study investigated the factors influencing quality of life among 501 infertile couples in Shiraz, Iran. Infertile couples with a shorter duration of infertility and male etiology had a higher quality of life. Lower levels of formal education, lower income, and prior unsuccessful treatment were associated with lower quality of life [21]. However, those three studies did not investigate the effects of irregularities in marital cohabitation on quality of life and sexual function among infertile men.

Scores indicating impairment on quality of life and sexual function inventories may be critical signs suggesting psychological distur- bances in infertile men [23]. Previous studies have analyzed several risk factors for sexual dysfunction and disturbed quality of life in infertile couples related to the burdens of infertility treatment, treatment failure, socioeconomic factors, and comorbidities associated with infertility, such as obesity and diabetes [24-27].

Another study investigated correlations between sexual dysfunction and quality of life among infertile couples. That study included 385 subjects (men and women), but without specifying how many men were included, and used the Iseng tool to measure self-esteem. A social support scaling test was used to assess social support, and quality of life was measured using the World Health Organization Quality of Life-BREF and FertiQoL questionnaires. The mean quality of life score in their study was $55.5 \pm 23.35$ among men, which is higher than that observed in the present study among infertile men with an irregular pattern of marital cohabitation (43.94 \pm 8.22$)$ and lower than that reported by infertile men with a regular pattern of marital cohabitation $(64.55 \pm 11.17)$. In that study, the researchers used the Lindberg questionnaire questionnaire to assess sexual satisfaction, and found that $3.5 \%$ were unsatisfied, $30.4 \%$ were partially satisfied, $56.8 \%$ were satisfied, and the rest were very satisfied. Lower scores were found among couples who had undergone previous treatment of infertility, and higher scores were found in couples with higher levels of education and income, as well as a shorter duration of infertility. In our study, we used IIEF-5 and PMEDT scores to evaluate erectile and ejaculatory function, respectively, and we found significant correlations between impairment in these scores and the duration of irregular marital cohabitation, regardless of other variables, such as infertility duration [20].

The present study showed the adverse effects of irregular patterns of marital cohabitation on quality of life and sexual function in infertile men. To the best of our knowledge, this study is the first to highlight the relationship between irregular patterns of marital cohabitation and impairments of both QoL and sexual function in infertile men. The mechanism by which irregular patterns of marital cohabitation affect QoL and sexual function may be through an instable physical relationship and emotional connection, along with the added stress induced by infertility and the requirements of infertility treatment. Further studies with detailed psychometric tests are required to analyze the mechanisms underlying these effects and to show which aspects of the marital relationship are more significantly impaired by irregular patterns of cohabitation.

\section{Conflict of interest}

No potential conflict of interest relevant to this article was reported. 


\section{ORCID}

Emad Abdelrhim Taha

Mohamed Sabry

Ahmed Hosny https://orcid.org/0000-0001-6549-2526

https://orcid.org/0000-0002-8206-2074

https://orcid.org/0000-0003-1472-327X

\section{Author contributions}

Conceptualization: EAT. Data Curation: EAT, AH, MS. Formal analysis: IFSA, HE. Methodology: EAT, MS. Project administration: EAT. Visualization: EAT, HE, MS. Writing - original draft: EAT, AH, IFSA. Writing - review \& editing: EAT, AH.

\section{References}

1. Boivin J, Bunting L, Collins JA, Nygren KG. International estimates of infertility prevalence and treatment-seeking: potential need and demand for infertility medical care. Hum Reprod 2007;22: 1506-12.

2. Chachamovich JR, Chachamovich E, Ezer H, Fleck MP, Knauth D, Passos EP. Investigating quality of life and health-related quality of life in infertility: a systematic review. J Psychosom Obstet Gynaecol 2010;31:101-10.

3. Maroufizadeh S, Karimi E, Vesali S, Omani Samani R. Anxiety and depression after failure of assisted reproductive treatment among patients experiencing infertility. Int J Gynaecol Obstet 2015;130: 253-6.

4. Lotti F, Corona G, Castellini G, Maseroli E, Fino MG, Cozzolino M, et al. Semen quality impairment is associated with sexual dysfunction according to its severity. Hum Reprod 2016;31:2668-80.

5. Strzemecka J, Pencula M, Owoc A, Szot W, Strzemecka E, Jablonski $M$, et al. The factor harmful to the quality of human life: shiftwork. Ann Agric Environ Med 2013;20:298-300.

6. Karabulut A, Demirtas O, Sonmez S, Karaca N, Gok S. Assessing factors associated with infertility using a couple-based approach. Int J Gynaecol Obstet 2017;137:96-8.

7. Teskereci G, Oncel S. Effect of lifestyle on quality of life of couples receiving infertility treatment. J Sex Marital Ther 2013;39:476-92.

8. Ghafar AA. Egyptians in revolt: the political economy of labor and student mobilizations 1919-2011. New York: Routledge; 2017.

9. Althof SE, McMahon CG, Waldinger MD, Serefoglu EC, Shindel AW, Adaikan PG, et al. An update of the International Society of Sexual Medicine's Guidelines for the Diagnosis and Treatment of Premature Ejaculation (PE). Sex Med 2014;2:60-90.

10. Boivin J, Takefman J, Braverman A. The Fertility Quality of Life (FertiQoL) tool: development and general psychometric proper- ties. Fertil Steril 2011;96:409-15.

11. World Health Organization. Laboratory manual for the examination of human semen and sperm-cervical mucus interactions. 5th ed. Cambridge: Cambridge University Press; 2010.

12. Boivin J, Takefman J, Braverman A. The fertility quality of life (FertiQoL) tool: development and general psychometric properties. Hum Reprod 2011;26:2084-91.

13. Rosen RC, Cappelleri JC, Smith MD, Lipsky J, Pena BM. Development and evaluation of an abridged, 5-item version of the International Index of Erectile Function (IIEF-5) as a diagnostic tool for erectile dysfunction. Int J Impot Res 1999;11:319-26.

14. Shamloul R, Ghanem $H$, Abou-zeid A. Validity of the Arabic version of the sexual health inventory for men among Egyptians. Int J Impot Res 2004;16:452-5.

15. Symonds T, Perelman MA, Althof S, Giuliano F, Martin M, May K, et al. Development and validation of a premature ejaculation diagnostic tool. Eur Urol 2007;52:565-73.

16. Smith NK, Madeira J, Millard HR. Sexual function and fertility quality of life in women using in vitro fertilization. J Sex Med 2015;12:985-93.

17. Valsangkar S, Bodhare T, Bele S, Sai S. An evaluation of the effect of infertility on marital, sexual satisfaction indices and health-related quality of life in women. J Hum Reprod Sci 2011;4:80-5.

18. Domar AD, Gross J, Rooney K, Boivin J. Exploratory randomized trial on the effect of a brief psychological intervention on emotions, quality of life, discontinuation, and pregnancy rates in in vitro fertilization patients. Fertil Steril 2015;104:440-51.

19. Goker A, Yanikkerem E, Birge O, Kuscu NK. Quality of life in Turkish infertile couples and related factors. Hum Fertil (Camb) 2018;21:195-203.

20. Keramat A, Masoomi SZ, Mousavi SA, Poorolajal J, Shobeiri F, Hazavhei SM. Quality of life and its related factors in infertile couples. J Res Health Sci 2014;14:57-63.

21. Namavar Jahromi B, Mansouri M, Forouhari S, Poordast T, Salehi A. Quality of life and its influencing factors of couples referred to an infertility center in Shiraz, Iran. Int J Fertil Steril 2018;11:293-7.

22. Hsu PY, Lin MW, Hwang JL, Lee MS, Wu MH. The fertility quality of life (FertiQoL) questionnaire in Taiwanese infertile couples. Taiwan J Obstet Gynecol 2013;52:204-9.

23. Wibowo E, Johnson TW, Wassersug RJ. Infertility, impotence, and emasculation: psychosocial contexts for abandoning reproduction. Asian J Androl 2016;18:403-8.

24. Alesi R. Infertility and its treatment: an emotional roller coaster. Aust Fam Physician 2005;34:135-8.

25. Peterson BD, Pirritano M, Block JM, Schmidt L. Marital benefit and coping strategies in men and women undergoing unsuccessful fertility treatments over a 5 -year period. Fertil Steril 
2011;95:1759-63.

26. Kocelak P, Chudek J, Naworska B, Bak-Sosnowska M, Kotlarz B, Mazurek $M$, et al. Psychological disturbances and quality of life in obese and infertile women and men. Int J Endocrinol 2012; 2012:236217.
27. Milazzo A, Mnatzaganian G, Elshaug AG, Hemphill SA, Hiller JE; Astute Health Study Group. Depression and anxiety outcomes associated with failed assisted reproductive technologies: a systematic review and meta-analysis. PLoS One 2016;11:e0165805. 